\title{
COHESIVE CRACK MODELING OF INFLUENCE OF SUDDEN CHANGES IN LOADING RATE ON CONCRETE FRACTURE
}

\author{
S. TANDON and $K$. T. FABER \\ Department of Materials Science and Engineering, Northwestern University, Robert R. McCormick \\ School of Engineering and Applied Science, Evanston, Illinois 60208-3108, U.S.A.
}

ZDENĚK P. BAŽANT and YUAN N. LI

Department of Civil Engineering, Northwestern University, Robert R. McCormick School of Engineering and Applied Science, Evanston, Illinois 60208-3108, U.S.A.

\begin{abstract}
The results of an experimental study of a sudden change in loading rate on the fracture behavior of normal- and high-strength concrete specimens of three different sizes are reported. Geometrically similar three-point bend specimens were subjected to either a sudden 1000 -fold increase or a 10 -fold decrease of the loading rate. It was observed that for a large increase of the loading rate, the post-peak softening can be reversed to hardening followed by a second peak of the stress-strain diagram. A sudden decrease of the loading rate initially causes a steeper softening slope of this diagram. The results are similar for normal and high strength concrete specimens. The viscoelastic cohesive crack model with the rate-dependent softening law is used to model the experimental results.
\end{abstract}

\section{INTRODUCTION}

ALTHOUGH CLASSICAL fracture mechanics is a rate-independent theory, the strength and fracture properties of Portland cement concrete and other cementitious materials, as well as many other brittle materials, depend on the loading rate [1-3]. One source of the rate sensitivity observed in brittle materials, such as concrete, is thermally activated crack growth $[4,5]$. The explanation of the rate sensitivity of crack growth is well known - the probability that the thermal vibration energy of an atom or molecule would exceed the activation energy barrier of the bond increases with the superimposed potential due to the applied stress. A second source of rate sensitivity is creep of the material in the bulk of the specimen, which alters the stress field near the crack tip [6-10]. The rate effects in concrete fracture have been thoroughly investigated at fast, dynamic loading rates, in which the time to reach peak load is less than $1 \mathrm{~s}$ [11-13]. Creep is negligible at fast loading rates but the inertial effects complicate the observed fracture behavior [14]. Creep effects, which have insufficient time to develop at fast, dynamic loading rates, dominate the fracture behavior at slow, static loading rates [15]. The fracture behavior of concrete structures with rates corresponding to the times to reach the peak load ranging up to many years is of great practical interest. This knowledge is needed to predict the long-term cracking and failure of large fracture-sensitive structures, such as concrete dams.

In a detailed study, Bažant and Gettu [16] investigated the rate effect in the static range with the time to peak load ranging from $1 \mathrm{~s}$ to 2.5 days. They showed the fracture toughness to decrease with a decreasing loading rate, similar to what had already been known for the dynamic range [17]. As a new, surprising result, the effective length of the fracture process zone was also found to decrease with a decreasing rate. From their study, the existence of a strong interaction between the fracture properties and the creep of concrete became clear. The results of Bažant and Gettu, which are limited to constant loading rates, have been modeled successfully by Bažant and $\mathrm{Li}[18]$ using a viscoelastic cohesive crack model with a rate-dependent softening law. Their model of fracture in a viscoelastic medium consists of a nonlinear version of the cohesive crack model, in which the process zone is considered to be of finite size. This model is applicable to the crack initiation stage; the crack-growth stage can be obtained as the asymptotic limit. The cohesive stress distribution in the process zone ahead of the actual crack tip is considered as 
the unknown and is solved by fracture analysis. To model typical tests, the solution is obtained under the condition of controlled crack-opening displacement. The details of this model, as well as general analyses of size effects and rate effects on the peak load, are presented by Bažant and Li [18].

The principal aim of this study is to examine the effect of a sudden increase or decrease of the loading rate on the fracture properties of normal- and high-strength concretes, both experimentally and theoretically. The experimental results are presented in the next section of the paper. Then, an extended cohesive crack model taking into account the viscoelasticity in the bulk material, as well as the time-dependent softening law of cohesive cracks is described briefly. Finally, the model is compared to the experimental data for sudden changes in the loading rate.

\section{EXPERIMENTAL DETAILS}

The materials used for this study-normal-strength concrete (NSC) and high-strength concrete (HSC)-were designed and mixed in the laboratory. The mix ratio (by weight) of the normal-strength concrete, was cement:sand:gravel:water $=1: 2: 2: 0.6$. The mix ratio of the high-strength concrete, by weight, was cement:sand:gravel:water:silica fume $=1: 2: 2: 0.3: 0.3$. The cement was ASTM Type I Portland cement and the sand was ASTM No. 2 sand. The maximum aggregate size in the mixes of normal- and high-strength concretes was $9.5 \mathrm{~mm} \mathrm{(3/8} \mathrm{in.).} \mathrm{In} \mathrm{addition,} 88.5 \mathrm{ml}$ of water reducing agent (W. R. Grace, Daracern-100) was added to one batch $\left(\sim 0.3 \mathrm{ft}^{3}\right)$ of the high-strength concrete mix. Silica fume (W. R. Grace, WRDA-19, microsilica) was used as a mineral admixture to modify and strengthen the interface between the aggregate particles and the matrix.

Geometrically similar single-edge notched beams of three different beam sizes were employed in the study. The specimens of three sizes were characterized by beam depths $d=38,76,152 \mathrm{~mm}$, designated $\mathrm{S}$-small, $\mathrm{M}$-medium and L-large. For all the beam specimens, the span-to-depth ratio was equal to 2.5 . The ratio of the initial notch length $a_{0}$ to specimen depth $D$ was 0.17 . The thickness of all specimens was constant $(38 \mathrm{~mm})$, which means the specimens were similar in two dimensions. This is preferable to three-dimensional similarity for reasons stated by Bažant and Pfeiffer [19]. All the specimens were compacted by rodding and vibration. During the first $24 \mathrm{~h}$ the specimens were left in the molds. Then the specimens were removed and cured in water until the time of testing. The notches were cut with a diamond band-saw and were $1.8-\mathrm{mm}$ wide. Companion cylinders of 76-mm diameter and 152-mm length also were cast. These cylinders were capped with a sulfur compound and were cured under water with the notched specimens. The cylinders were tested in compression after 28 days of curing. The normal-strength concrete cylinders failed at an average maximum compressive stress of $46.4 \mathrm{MPa}$, with a standard deviation of $5.4 \%$. The high-strength concrete cylinders had an average compressive strength of $73.2 \mathrm{MPa}$, with a standard deviation of $3.3 \%$.

The notched beam specimens were tested under crack-mouth opening displacement (CMOD) control in a $89 \mathrm{kN}$ load frame with an MTS (MTS 810, Materials Testing Systems, Minneapolis, MN) closed-loop control system. During each test the load, cross-head displacement and CMOD were measured. The CMOD, which is the basic indicator of the crack growth in the specimen, was measured by a COD clip gage (MTS, COD gage 632.13B-20). All the geometrically similar three-point-bend specimens of all sizes were subjected to either a sudden 1000 -fold increase or a 10 -fold decrease in the loading rate of the post-peak region. The machine response to such a rate change is nearly instantaneous, with a delay of less than $0.5 \mathrm{~s}$ after the electronic signal, which is insignificant compared to the duration of the test.

\section{Summary of experimental results}

These experiments show an overall consistent picture for normal- and high-strength concretes, similar to the preliminary results reported by Bažant et al. [20] for normal-strength concrete only. For normal- and high-strength concrete specimens tested under similar loading histories, the results can be summarized as follows.

1. A sudden increase of the loading rate reverses the post-peak softening to post-peak hardening followed by a second peak. This is illustrated for a large-size, normal-strength concrete specimen in 
Fig. 1(a). The initial CMOD rate was $\sim 10^{-5} \mathrm{~mm} / \mathrm{sec}$, and after the peak load $P_{1}$ at $P_{c}$, the CMOD rate was suddenly increased 1000 -fold to $\sim 10^{-2} \mathrm{~mm} / \mathrm{sec}$. The post-peak softening curve is seen to reverse to hardening, with a second peak at $\sim 105 \% P_{1}$. The second peak may be higher or lower than the first peak at the previous slow rate of loading, depending on the ratio of rate increase and the magnitude of load decrease prior to the increase of loading rate. Figure 1(b) shows the response to a sudden increase in loading rate after a load drop to $\sim 90 \%$ of the previous peak load in a large-size, high-strength concrete specimen.

2. After a sudden decrease of loading rate, the slope of the measured load-CMOD diagram suddenly becomes steeper, but later the previous slope is resumed again. The specimens were initially at $\sim 10^{-5} \mathrm{~mm} / \mathrm{sec}$ and, in the post-peak regime, the CMOD rate was suddenly decreased to $\sim 10^{-6} \mathrm{~mm} / \mathrm{sec}$. The typical results for normal- and high-strength concrete specimens are shown in Fig. 2(a) and (b), respectively. The sudden decrease in the loading rate was accompanied by an almost instantaneous drop in load, followed by a conventional post-peak softening response.

3. The effects of the sudden decrease or increase in the loading rates are similar for different geometrically similar sized notched specimens of normal- as well as high-strength concrete. The test results obtained on normal-strength concrete and high-strength concrete beam specimens of the three sizes are summarized in Tables 1 and 2, respectively.
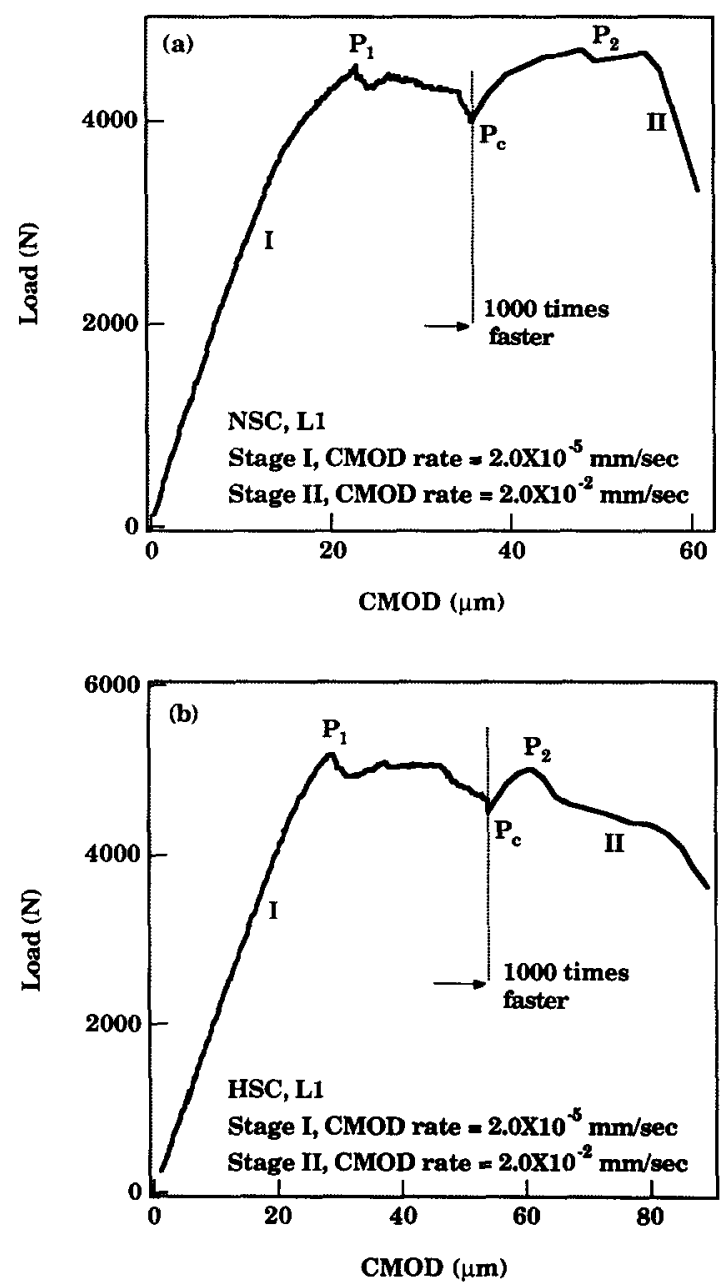

Fig. 1. (a) Load vs CMOD response of a large-size, normal-strength concrete beam specimen for a 1000 -fold rate increase at point $P_{c}$, after a load drop to $\sim 90 \% P_{1}$. (b) Load vs CMOD response of a largesize, high-strength concrete beam specimen for a 1000 -fold rate increase at point $P_{c}$, after a load drop to $\sim 90 \% P_{1}$. 

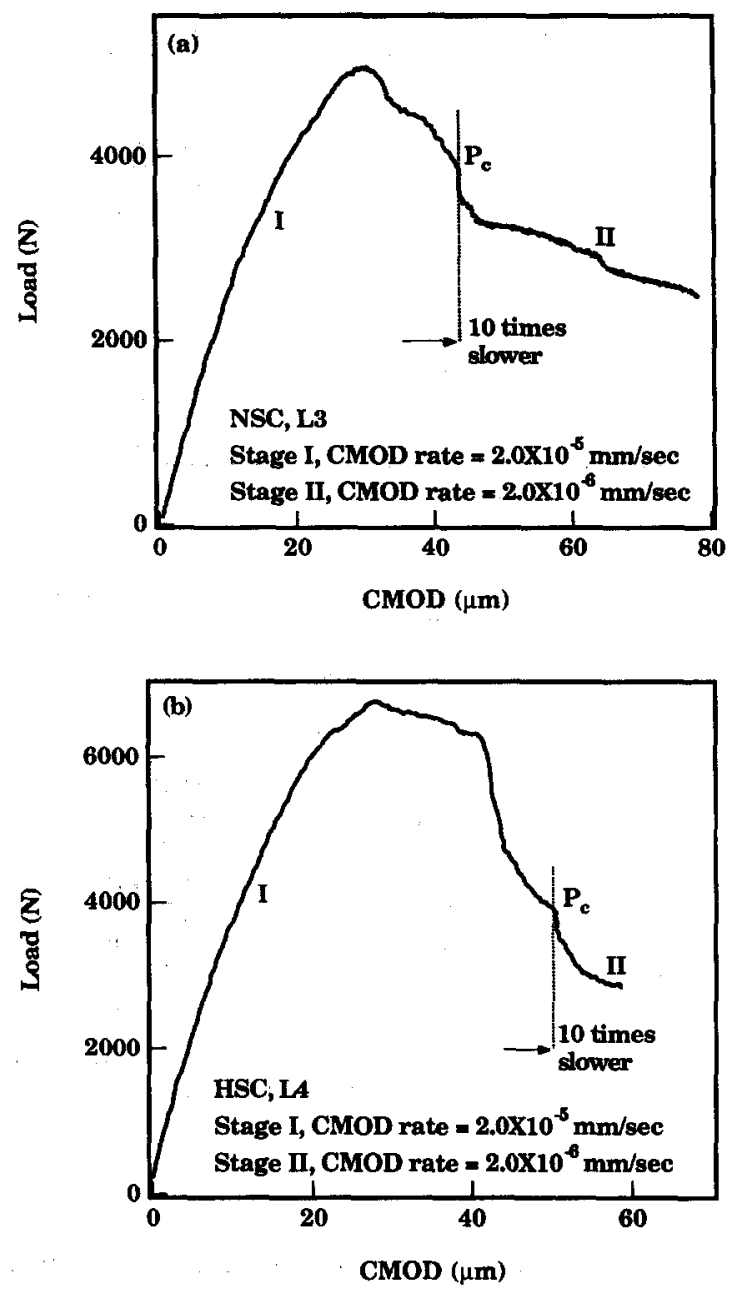

Fig. 2. (a) Load vs CMOD response of a large-size, normal-strength concrete beam specimen for a 10 -fold rate decrease at point $P_{c}$, after a load drop to $\sim 70 \% P_{1}$. (b) Load vs CMOD response of a large-size, high-strength concrete beam specimen for a 10 -fold rate decrease at point $P_{c}$, after a load drop to $\sim 70 \% P_{1}$.

\section{VISCOELASTIC COHESIVE CRACK MODEL WITH RATE-DEPENDENT SOFTENING}

For an elastic structure such as the three-point bend beam shown in Fig. 3, the crack-opening displacement $w$ and the cohesive (crack-bridging) stress $\sigma$ must satisfy the following compatibility condition:

$$
w(x)=-\int_{a_{0}}^{a} \bar{C}^{w \sigma}\left(x, x^{\prime}\right) \sigma\left(x^{\prime}\right) \mathrm{d} x^{\prime}+\bar{C}^{w P}(x) P,
$$

where $\bar{C}^{w \sigma}\left(x, x^{\prime}\right)$ and $\bar{C}^{w P}(x)$ are the geometric compliance functions of the elastic specimen, that is, compliances for a unit value of elastic modulus $E$. These compliances exhibit the proper symmetry according to linear elastic reciprocity; $a_{0}$ is the notch length, $a$ is the total cohesive crack length including the process zone length, and $P$ is the load. The problem considered here is two-dimensional and the structure is assumed to be of unit thickness $(b=1)$.

When the material is viscoelastic, the deformation depends on the loading history, and the elastic relation described in eq. (1) must be generalized according to the principle of superposition and the elastic-viscoelastic analogy [21]. For an aging linearly viscoelastic material (with a constant creep 
Table 1. Data on fracture tests on normal-strength concrete beams

\begin{tabular}{|c|c|c|c|c|c|c|}
\hline \multirow[b]{2}{*}{ Specimen $\dagger$} & \multirow[b]{2}{*}{$\begin{array}{c}\text { Age } \\
\text { (days) }\end{array}$} & \multirow[b]{2}{*}{$\begin{array}{c}\text { Rate } \\
(\mathrm{mm} / \mathrm{sec})\end{array}$} & \multirow{2}{*}{$\begin{array}{c}\text { First stage } \\
\text { Load at rate change, } \\
P_{c}(\mathrm{~N})\end{array}$} & \multirow[b]{2}{*}{$\begin{array}{c}\text { Peak load, } \\
P_{1}(\mathrm{~N})\end{array}$} & \multicolumn{2}{|c|}{ Second stage } \\
\hline & & & & & $\begin{array}{c}\text { Rate } \\
(\mathrm{mm} / \mathrm{sec})\end{array}$ & $\begin{array}{c}\text { Peak load, } \\
\boldsymbol{P}_{2}(\mathrm{~N})\end{array}$ \\
\hline$\overline{\mathrm{S} 2}$ & 35 & $1.1 \times 10^{-5}$ & 1600 & $91 \% P_{1}$ & $1.1 \times 10^{-2}$ & $109 \% P_{1}$ \\
\hline M1 & 28 & $1.5 \times 10^{-5}$ & 2837 & $90.3 \% P_{1}$ & $1.5 \times 10^{-2}$ & $100 \% P_{1}$ \\
\hline L1 & 36 & $2.0 \times 10^{-5}$ & 4456 & $90.3 \% P_{1}$ & $2.0 \times 10^{-2}$ & $105 \% P_{1}$ \\
\hline M2 & 28 & $1.5 \times 10^{-5}$ & 2408 & $64.2 \% P_{1}$ & $1.5 \times 10^{-2}$ & $69 \% P_{1}$ \\
\hline $\mathrm{L} 2$ & 36 & $2.0 \times 10^{-5}$ & 4874 & $71.2 \% P_{1}$ & $2.0 \times 10^{-2}$ & $80 \% P_{1}$ \\
\hline L4 & 37 & $2.0 \times 10^{-4}$ & 5054 & $78.2 \% P_{1}$ & $2.0 \times 10^{-2}$ & $85.5 \% P_{1}$ \\
\hline L6 & 37 & $2.0 \times 10^{-5}$ & 4596 & $29.7 \% P_{1}$ & $2.0 \times 10^{-2}$ & $32.8 \% P_{1}$ \\
\hline M5 & 30 & $1.5 \times 10^{-5}$ & 2320 & $71.7 \% P_{1}$ & $1.5 \times 10^{-6}$ & - \\
\hline L3 & 36 & $2.0 \times 10^{-5}$ & 4977 & $73.4 \% P_{1}$ & $2.0 \times 10^{-6}$ & - \\
\hline
\end{tabular}

†S-small $(d=38 \mathrm{~mm}), \mathrm{M}-$ medium $(d=76 \mathrm{~mm}), \mathrm{L}-$ large $(d=152 \mathrm{~mm})$.

Table 2. Data on fracture tests on high-strength concrete beams

\begin{tabular}{lcccccc}
\hline Speciment & $\begin{array}{c}\text { Age } \\
\text { (days) }\end{array}$ & $\begin{array}{c}\text { Rate } \\
(\mathrm{mm} / \mathrm{sec})\end{array}$ & $\begin{array}{c}\text { First stage } \\
\text { Load at rate change, } \\
\boldsymbol{P}_{c}(\mathrm{~N})\end{array}$ & $\begin{array}{c}\text { Peak load, } \\
\boldsymbol{P}_{1}(\mathrm{~N})\end{array}$ & $\begin{array}{c}\text { Recond stage } \\
\text { (mm/sec) }\end{array}$ & $\begin{array}{c}\text { Peak load, } \\
\boldsymbol{P}_{2}(\mathrm{~N})\end{array}$ \\
\hline S1 & 60 & $1.1 \times 10^{-5}$ & 1326 & $92 \% P_{1}$ & $1.1 \times 10^{-2}$ & $113 \% P_{1}$ \\
M1 & 59 & $1.5 \times 10^{-5}$ & 2424 & $93.1 \% P_{1}$ & $1.5 \times 10^{-2}$ & $107 \% P_{1}$ \\
L1 & 64 & $2.0 \times 10^{-5}$ & 5183 & $86.8 \% P_{1}$ & $2.0 \times 10^{-2}$ & $96.7 \% P_{1}$ \\
S2 & 60 & $1.1 \times 10^{-5}$ & 1292 & $68.4 \% P_{1}$ & $1.1 \times 10^{-2}$ & $79.4 \% P_{1}$ \\
M2 & 59 & $1.5 \times 10^{-5}$ & 3307 & $68.5 \% P_{1}$ & $1.5 \times 10^{-2}$ & $78.8 \% P_{1}$ \\
L2 & 64 & $2.0 \times 10^{-5}$ & 5089 & $68.7 \% P_{1}$ & $2.0 \times 10^{-2}$ & $77.2 \% P_{1}$ \\
M3 & 60 & $1.5 \times 10^{-5}$ & 3176 & $32.1 \% P_{1}$ & $1.5 \times 10^{-2}$ & $36.7 \% P_{1}$ \\
L3 & 64 & $2.0 \times 10^{-5}$ & 5961 & $30 \% P_{1}$ & $2.0 \times 10^{-2}$ & $33.4 \% P_{1}$ \\
M4 & 60 & $1.5 \times 10^{-9}$ & 2982 & $73.4 \% P_{1}$ & $1.5 \times 10^{-6}$ & - \\
L4 & 65 & $2.0 \times 10^{-5}$ & 6772 & $56.2 \% P_{1}$ & $2.0 \times 10^{-6}$ & - \\
\hline
\end{tabular}

$\lceil\mathrm{S}-\operatorname{small}(d=38 \mathrm{~mm}), \mathrm{M}$-medium $(d=76 \mathrm{~mm})$, L-large $(d=152 \mathrm{~mm})$.

Poisson's ratio equal to the elastic Poisson's ratio), this generalization leads to the compatibility condition [18]:

$$
w(x, t)=-\int_{a_{0}}^{a} \bar{C}^{w \sigma}\left(x, x^{\prime}\right) \sigma_{\mathrm{eff}}\left(x^{\prime}, t\right) \mathrm{d} x^{\prime}+\bar{C}^{w P}(x) P_{\mathrm{ent}}(t)
$$

where the effective cohesive stress and the effective load are, respectively,

$$
\sigma_{\mathrm{eff}}(x, t)=\int_{t_{\mathrm{o}}(x)}^{t} J\left(t, t^{\prime}\right) \sigma\left(x, \mathrm{~d} t^{\prime}\right)
$$

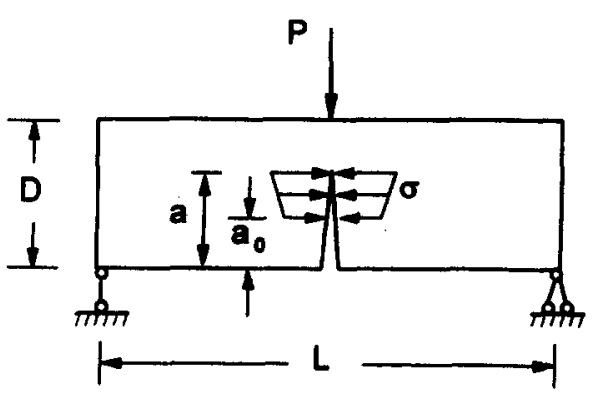

Fig. 3. Three-point bend elastic structure, $\sigma$ is the bridging stress.

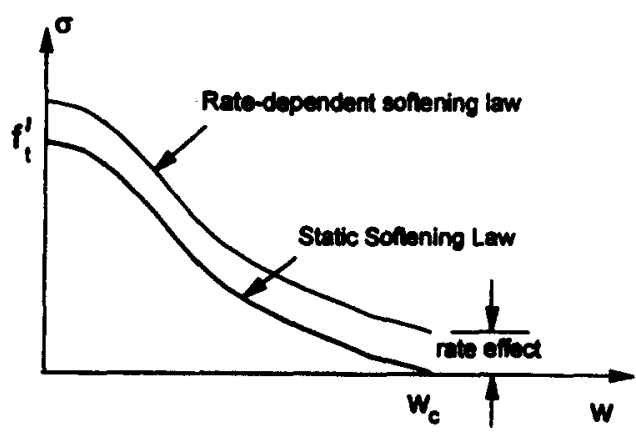

Fig. 4. Static and rate-dependent softening law. 
and

$$
P_{\mathrm{eff}}(t)=\int_{t_{0}}^{t} J\left(t, t^{\prime}\right) \mathrm{d} P\left(t^{\prime}\right)
$$

Here $t_{0}$ is the time at which the load is first applied, $J\left(t, t^{\prime}\right)$ is the compliance function of the material representing the strain at age $t$ caused by a unit uniaxial stress acting since age $t_{0}$. For a continuous stress history, $\sigma\left(x, \mathrm{~d} t^{\prime}\right)=\left[\partial \sigma(x, t) / \partial t^{\prime}\right] \mathrm{d} t^{\prime}$, and in the case of a jump at time $t_{1}$, the part of the integral in eq. (3) from $t_{1}^{-}$to $t_{1}^{+}$is $J\left(t, t_{1}\right)\left[\sigma\left(x, t_{1}^{+}\right)-\sigma\left(x, t_{1}^{-}\right)\right]$.

For the present load durations, the compliance function can be approximated by the double-power law [22]

$$
J\left(t, t^{\prime}\right)=\frac{1}{\beta}\left[1+\phi\left(t^{-m}+\alpha\right)\left(t-t^{\prime}\right)^{n}\right]
$$

where $\phi=3-6, \alpha=0.05, m=1 / 3, n=1 / 8$ and $\beta$ is typically $1.5-2$. Note that the instantaneous modulus $E_{0}=1 / J\left(t, t^{\prime}\right)\left(t^{\prime}=1\right)$ according to eq. (5) is 1.5-2 times the standard static initial Young's modulus $E$, which corresponds to a loading duration of approximately 0.1 day. These values are given under the condition that the time is measured in days.

Based on the concept of activation energy, Bažant [23] proposed and Bažant and Li [18] used the following rate-dependent softening law:

$$
w=g\left[\sigma-\kappa a \sinh \left(\frac{\dot{w}}{\dot{w}_{0}}\right) f_{t}^{\prime}\right]
$$

where $\dot{w}_{0}$ is the reference crack-opening rate, $\kappa$ is a dimensionless empirical parameter, usually in the range of $0.01-0.05$ for concrete. The parameter $\kappa$ determines the overall sensitivity of the softening law to a change of crack-opening rate, while the parameter $\dot{w}_{0}$ determines the lower limit of the loading rate, beyond which the rate-dependence becomes significant. The function $g$ is the static softening law, and asinh is the inverse of the hyperbolic sine function. As shown in Fig. 4, this form of the softening law is simply a parallel shift of the static softening law, with the shift distance dependent on the crack-opening rate.

Consequently, for the viscoelastic material, the crack compatibility condition can be written as

$$
g\left[\sigma(x, t)-\kappa \operatorname{asinh}\left(\frac{\dot{w}(x, t)}{\dot{w}_{0}}\right) f_{t}^{\prime}\right]=-\int_{a_{0}}^{a} \bar{C}^{w \sigma}\left(x, x^{\prime}\right) \sigma_{e f f}\left(x^{\prime}, t\right) \mathrm{d} x^{\prime}+\bar{C}^{w P}(x) P_{\mathrm{eff}}(t) .
$$

From eq. (2), the crack-opening rate can be expressed as

$$
\dot{w}(x, t)=-\int_{a_{0}}^{a} \bar{C}^{w \sigma}\left(x, x^{\prime}\right) \dot{\sigma}_{\mathrm{eff}}\left(x^{\prime}, t\right) \mathrm{d} x^{\prime}+\bar{C}^{w P}(x) \dot{P}_{\mathrm{eff}}(t) .
$$

The time derivative of the effective cohesive stress in this equation is expressed as

$$
\dot{\sigma}_{\mathrm{eff}}(x, t)=\frac{1}{E(t)} \dot{\sigma}(x, t)+\int_{t_{0}(x)}^{t} \frac{\partial J\left(t, t^{\prime}\right)}{\partial t} \frac{\partial \sigma\left(x, t^{\prime}\right)}{\partial t^{\prime}} \mathrm{d} t^{\prime},
$$

where $E(t)=1 / J(t, t)=$ elastic modulus at age $t$. In eq. (9), the first term is the elastic stress rate and the second term is due to creep. The latter represents the influence of the viscoelasticity of the bulk material on the response in the process zone. Even if the stress rate is zero, the effective stress rate is not zero because of the viscoelastic term. A similar expression can be written for the time derivative of the effective load. 

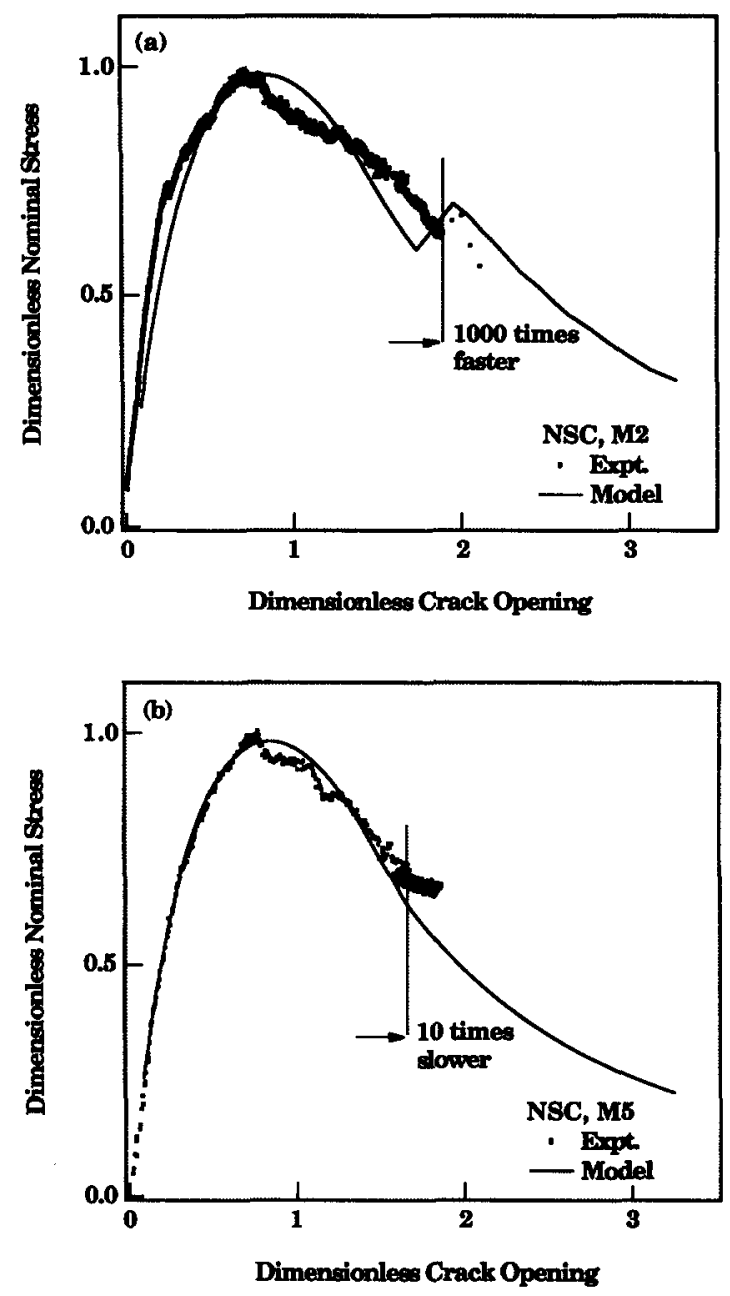

Fig. 5. (a) Experimental and predicted load vs CMOD response of a medium-size, normal-strength concrete beam specimen for a 1000-fold rate increase. (b) Experimental and predicted load vs CMOD response of a medium-size, normal-strength concrete beam specimen for a 10-fold rate decrease.

\section{PREDICTION OF MECHANICAL RESPONSE FOR A SUDDEN CHANGE IN LOADING RATE}

The finite element method is used to obtain the compliance functions for different crack lengths. The crack length $a$ can therefore assume only discrete values in accordance with the mesh used in the calculation. Since the focus of the analysis is on crack initiation rather than steady-state crack propagation, it is natural to use one parameter, the crack length $a$. Time $t$ and the cohesive stress $\sigma$ are the further unknowns. If the test is run under load control, the time history $P(t)$ is given. If the test is run under displacement control, the crack-opening displacement $w$ described in eq. (2) must be used to solve for the applied load, the cohesive stress and the time required for the given crack-length increment. The crack length is prescribed to vary in discrete steps, taking discrete values of $a_{i}$ given by the mesh nodes $\left(i=0,1, \ldots q ; a_{i}<a_{i+1}\right)$. The stress and the applied load are assumed to vary linearly within each step from $t_{i}$ to $t_{i+1}$, where $t_{i}$ is the time when the basic equations are satisfied. It is most effective to use the rate of cohesive stress and the rate of load as the basic unknowns to solve for. The total stress, as well as the effective stress, can be expressed in terms of these rate unknowns approximating the loading integrals by sums. A more detailed description of the numerical technique is discussed in a separate paper [24].

To compare experimental results with the predicted results, all variables are expressed in 
nondimensional form. The applied load $P$ is usually characterized in terms of nominal stress $\sigma_{\mathrm{N}}$, defined as

$$
\sigma_{\mathrm{N}}=\frac{7.5 P}{b D}
$$

where $b$ is the thickness of the beam and $D$ is the beam depth. The nominal stress $\sigma_{\mathrm{N}}$ is then divided by the tensile strength of concrete, $f^{\prime}{ }^{\prime}$, to get a dimensionless nominal stress. The measured CMOD is divided by the threshold value of the crack-opening displacement $w_{c}$ to provide the dimensionless crack opening. The characteristic size, representing the beam depth $D$ is divided by the material length $l_{0}$ to make it dimensionless. The material length is defined as

$$
l_{0}=\frac{E G_{f}}{\left(f_{i}^{\prime}\right)^{2}}
$$

where $E$ is the Young's modulus, $G_{f}$ is the fracture energy and $f^{\prime}$, is the tensile strength of the material. For a material following a linear softening law,

$$
l_{0}=\frac{E w_{c}}{2 f_{t}^{\prime}} .
$$

In this study, the following material parameters are used, $f^{\prime}{ }_{t}=7.2 \times 10^{6} \mathrm{~N} / \mathrm{m}^{2}, l_{0}=0.1 \mathrm{~m}, w_{c}$ $=24 \mu \mathrm{m}$. For the rate-dependent softening law [eq. (6)] the parameters are chosen as $\kappa=0.03$ and the reference opening displacement rate $\dot{w}_{0}=100 / \mathrm{min}$. It is assumed that the material follows a linear softening law given by

$$
g(\sigma)=w_{c}\left(1-\frac{\sigma}{f_{i}^{\prime}}\right)
$$

The constants required for the compliance function are $\phi=3.926$ and $\beta=1.8$. These values are selected for a beam of a certain nondimensional size under the condition that the effective Young's modulus at the peak load be equal to the static Young's modulus when the loading rate produces a time to peak of approximately $10 \mathrm{~min}$. Since the curing time influences the strength of concrete, the compressive strength of concrete is variable. For ages over 28 days, the strength evolution can be approximately estimated from the ACI formula:

$$
\frac{\left(f_{c}^{\prime}\right)_{t}}{\left(f_{c}^{\prime}\right)_{28}}=\frac{t}{0.85 t+4.2}
$$

where $t$ is the age of the specimen in days and $\left(f^{\prime}{ }_{c}\right)_{28}$ is the standard cylindrical compressive strength of concrete after 28 days of curing. It is assumed here that the ratio of tensile strength of concrete at any age to its value at 28 days can be calculated using the same formula.

\section{Results for sudden change in loading rate}

The experimental results for a sudden change in loading rate for the medium size $(D=76 \mathrm{~mm})$ specimens of normal-strength concrete are compared with the predicted results in Fig. 5 . The ratio of the load at which the loading rate was changed to the first peak load is specified for the calculations by the model. The predicted results are in good agreement with the experimental results for these specimens. Figure 6 shows the comparison of the experimental results to the predicted results for a sudden change in loading rate for medium-size high-strength concrete specimens. From these results it is clear that the viscoelastic cohesive crack model with rate-dependent softening can describe the observed response of both normal- and high-strength concretes for a sudden change in loading rate in the softening branch.

For a sudden change in loading rate of high-strength concrete specimens of all three sizes, the comparison is made in Fig. 7. The predicted results are in close agreement with the experimental results for these specimens. The present model, therefore, is able to describe the behavior for all three different sizes of geometrically similar specimens too.

When the predicted results are compared with the experimental results, it is seen that some of the experimental response curves exhibit small local peaks before the overall peak is reached. In some 
of the specimens one can see a relatively flat region occurring after the first peak. These two phenomena are not systematic and are probably caused by random effects associated with specimen heterogeneity.

The ratio of the two peak loads, $\boldsymbol{P}_{2} / \boldsymbol{P}_{1}$, is shown in Fig. 8 as a function of $\boldsymbol{P}_{c} / \boldsymbol{P}_{1}$. The calculated results for normal- and high-strength concrete specimens can be represented by a straight line, which is virtually independent of the specimen size. The experimentally observed results agree very well with the results predicted by this model. Also, this result is very close to the result of Bažant and Jirásek [24]. As shown by Bažant and $\mathrm{Li}[18]$, the slope of this curve can be controlled by the parameter $\kappa$ or $\dot{w}_{0}$, or both. Good agreement with experimental data indicates that good values of the fit parameters of the model have been identified.

\section{CONCLUSIONS}

1. The viscoelastic cohesive crack model with rate-dependent softening can predict the response to sudden loading-rate changes observed in the laboratory. For a sudden increase in the loading rate, a second peak, lower or higher than the first peak, is observed on the stress-strain diagram. For a sudden decrease in the loading rate, the slope of the diagram sharply decreases and the response approaches the load-CMOD curve for the lower rate. The experimental results for both the normaland high-strength concretes are in good agreement with the predicted results.
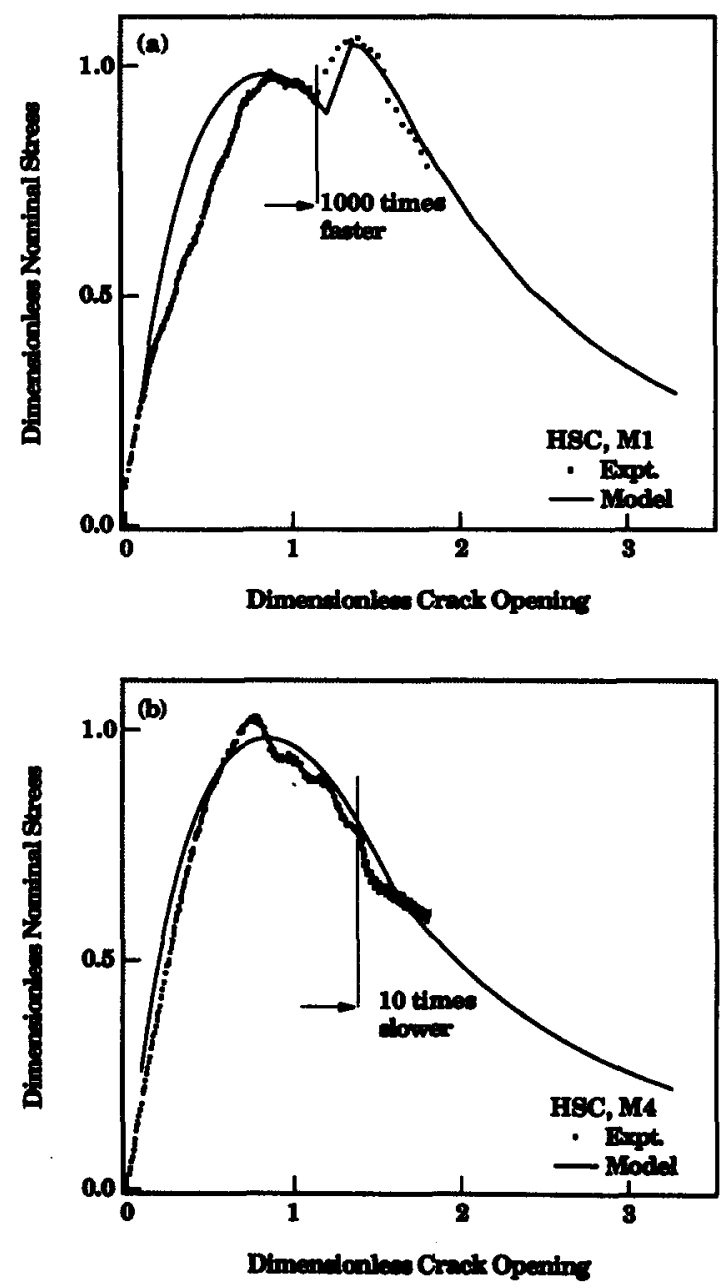

Fig. 6. (a) Experimental and predicted load vs CMOD response of a medium-size, high-strength concrete beam specimen for a 1000-fold rate increase. (b) Experimental and predicted load vs CMOD response of a medium-size, high-strength concrete beam specimen for a 10-fold rate decrease. 

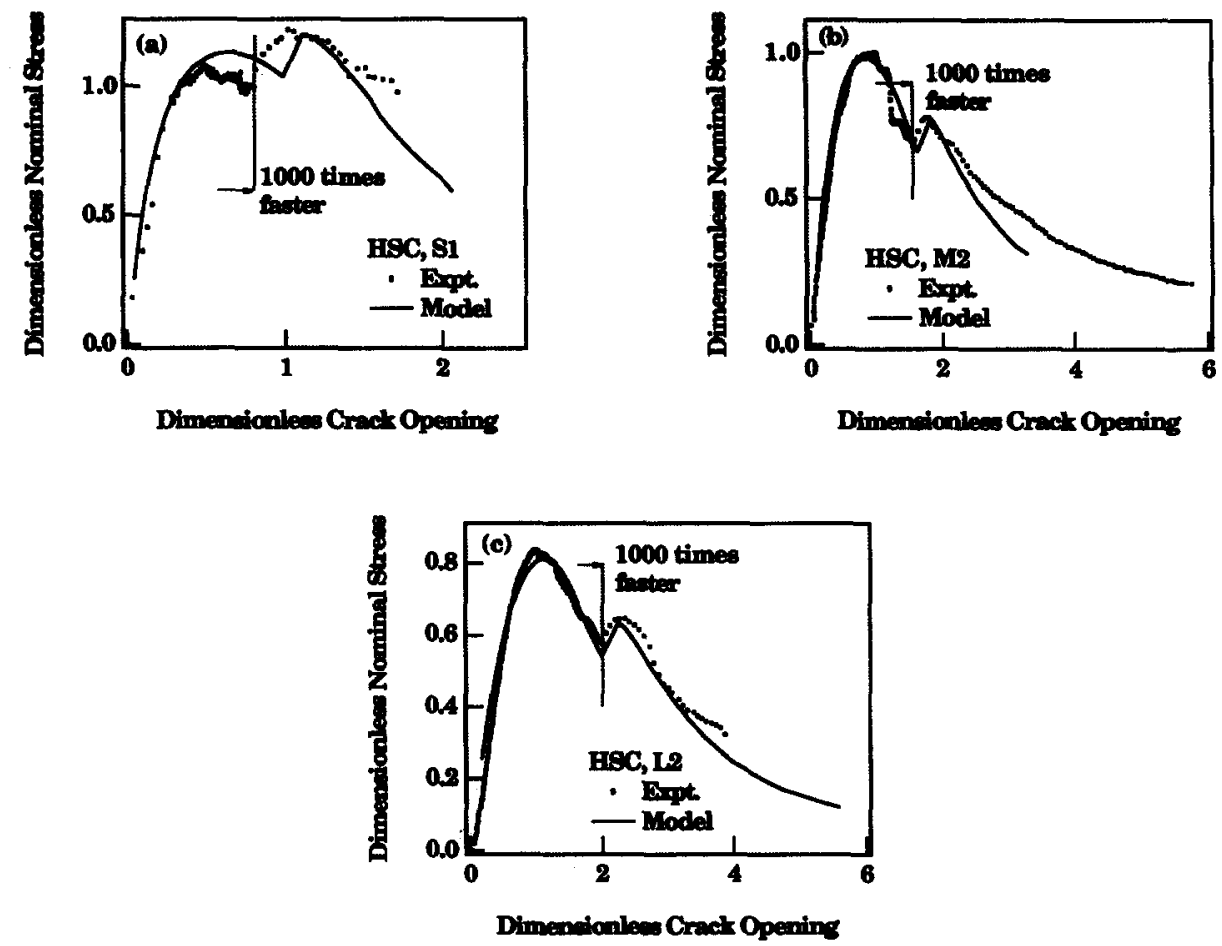

Fig. 7. (a) Experimental and predicted load vs CMOD response of a small-size, high-strength concrete beam specimen for a 1000 -fold rate increase. (b) Experimental and predicted load vs CMOD response of a medium-size, high-strength concrete beam specimen for a 1000 -fold rate increase. (c) Experimental and predicted load vs CMOD response of a large-size, high-strength concrete beam specimen for a 1000 -fold rate increase.

2. This model can predict the experimental results for very different sizes of geometrically similar specimens.

3. The ratio of the second peak load for the increased loading rates to the first peak load for the initial loading rate, as a function of the ratio of the load at which the rate is increased to the first peak load, is independent of the specimen size. This is also predicted by viscoelastic cohesive crack model. The second peak appears to be governed mainly by the rate-dependence of the softening law.

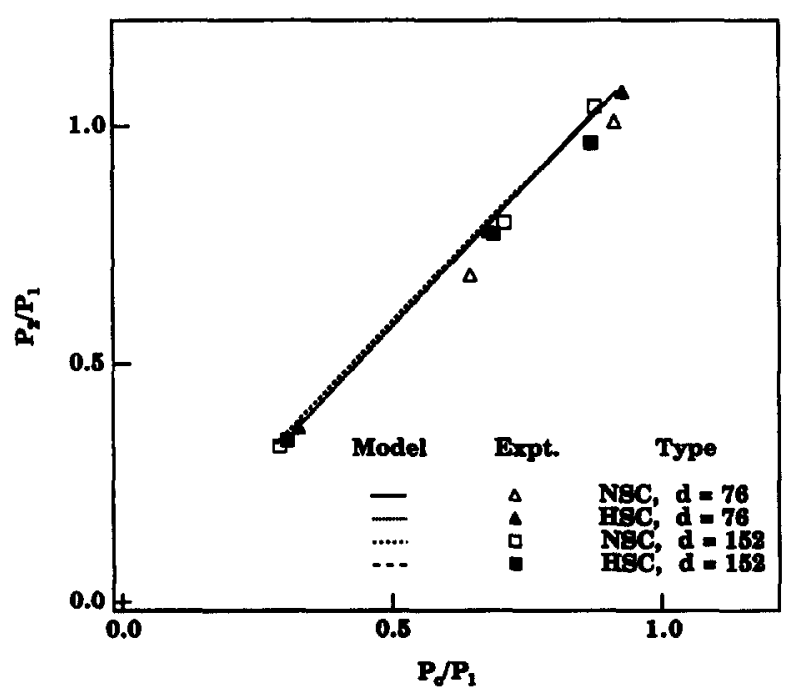

Fig. 8. Comparison of predicted rate effect on the second peak load with the experimental data. 
Acknowledgements - This experimental work was supported by the Center for Advanced Cement-Based Materials at Northwestern University. Further support for the theoretical formulation has been obtained under NSF grant MSS 9114476 for Northwestern University.

\section{REFERENCES}

[1] D. McHenry and J. J. Shideler, ASTM Special Technical Publication No. 185. Philadelphia, pp. 72-82 (1956).

[2] S. Mindess and J. S. Nadeau, Am. Ceram. Soc. Bull. 56, 429-430 (1977).

[3] S. Mindess, Application of Fracture Mechanics to Cementitious Composites (Edited by S. P. Shah), NATO-ARW, Northwestern University, U.S.A. Martinus Nijhoff, Dordrecht, The Netherlands, pp. 617-636 (1984).

[4] R. J. Charles, J. appl. Phys. 29, 1549-1560 (1958).

[5] A. G. Evans, Int. J. Fracture 10, 251-259 (1974).

[6] F. H. Wittman and J. Zaitsev, Mechanical Behawior of Materials, Vol. IV. The Society of Materials Science, Japan, pp. 84-95 (1972).

[7] J. W. Zaitsev and F. H. Wittman, Cem. Concr. Res. 3, 389-395 (1973).

$[8]$ S. P. Shah and S. Chandra, $A C I J .65,770-781$ (1968).

[9] S. P. Shah and S. Chandra, $A C I J .67,816-825$ (1970).

[10] Z.-G. Liu, S. E. Swartz, K. K. Hu and Y.-C. Kan, Fracture of Concrete and Rock: Recent Developments (Edited by S. P. Shah, S. E. Swartz and B. I. G. Barr), Int. Conf., Cardiff, U.K. Elsevier Applied Science, London, pp. 577-586 (1989).

[11] Cement-based Composites: Strain Rate Effects on Fracture (Edited by S. Mindess and S. P. Shah). Boston, p. 270 (1986).

[12] H. W. Reinhardt, Application of Fracture Mechanics to Cementitious Composites (Edited by S. P. Shah), NATO-ARW, Northwestern University, U.S.A. Martinus Nijhoff, Dordrecht, The Netherlands, pp. 559-590 (1984).

[13] C. A. Ross, Toughening Mechanisms in Quasi-brittle Materials (Edited by S. P. Shah), NATO-ARW, Northwestern University, U.S.A. Kluwer Academic, Dordrecht, The Netherlands, pp. 577-596 (1984).

[14] W. Suaris and S. P. Shah, J. Struct. Engng 109, 1727-1741 (1983).

[15] K. Newman, Cement and Concrete Association-Symposium on Concrete Quality, London, pp. 120-138 (1964).

[16] Z. P. Bažant and R. Gettu, ACI Mat. J. 89, 456-468 (1992).

[17] F. H. Wittmann, Application of Fracture Mechanics to Cementitious Composites (Edited by S. P. Shah), NATO-ARW, Northwestern University, U.S.A. Martinus Nijhoff, Dordrecht, The Netherlands, pp. 593-615 (1984).

[18] Z. P. Bažant and Y. N. Li, Int. J. Fracture (in press).

[19] Z. P. Bažant and P. A. Pfeiffer, ACI Mat. J. 84, 463-480 (1987).

[20] Z. P. Bažant, W.-H. Gu and K. T. Faber, ACI Mat. J. 92, 3-9 (1995).

[21] Z. P. Bažant, Computer Modelling of Concrete Structures (Edited by H. Mang, N. Bicanic and R. De Borst), Euro-C 1994 International Conf., pp. $461-480$ (1994).

[22] Z. P. Bažant and L. Cedolin, Stability of Structures: Elastic, Inelastic, Fracture and Damage Theories. Oxford University Press, New York (1991).

[23] Z. P. Bažant, Creep and Shrinkage of Concrete (Edited by Z. P. Bažant and I. Carol), Fifth International RILEM Symposium, 1993, pp. 291-307.

[24] Z. P. Bažant and M. Jirásek, R-curve modeling of rate and size effects in quasibrittle fracture. Int. J. Fracture 62, 355-373 (1993). 